\title{
Pharmacological properties, functional alterations, and gene expression of muscarinic receptors in young and old type 2 Goto-Kakizaki diabetic rat bladders
}

Motoaki Saito, Shin-ichi Okada, Emi Kazuyama, Itaru Satoh, Yukako Kinoshita, and Keisuke Satoh

Department of Pathophysiological and Therapeutic Science, Division of Molecular Pharmacology, Tottori University Faculty of Medicine, Yonago, Japan

Key Words: urinary bladder, Goto-Kakizaki (GK) rat, cystopathy, muscarinic $\mathrm{M}_{2}$ receptor, muscarinic $\mathrm{M}_{3}$ receptor, mRNA

Running head: Type 2 diabetic rat bladder

Correspondence:

Motoaki Saito, MD, PhD

Department of Pathophysiological and Therapeutic Science, Division of Molecular Pharmacology, Tottori University Faculty of Medicine, 86 Nishimachi, Yonago 683-8503, Japan

Tel: $\quad+81-859-38-6163$
Fax: $\quad+81-859-38-6160$
E-mail: saitomo@grape.med.tottori-u.ac.jp 


\begin{abstract}
Purpose: We investigated pharmacological properties, functional alterations and gene expressions of the muscarinic receptor system in young and old Goto-Kakizaki (GK) rat bladders.
\end{abstract}

Materials and methods: Twelve- and 70-week-old male GK rats and age-matched male Wistar rats were used in this study. The bladder functions were estimated by voiding behavior studies, cystometric studies, and functional studies using $\mathrm{KCl}$, carbachol, and various concentrations of subtype selective muscarinic antagonists, i.e., atropine, pirenzepine, methoctramine, and 4-diphenylacetoxy-N-methylpiperidine methiodide (4-DAMP). The participation levels of $\mathrm{M}_{2}$ and $\mathrm{M}_{3}$ receptor mRNAs in the bladder were investigated by real-time PCR.

Results: In the voiding behavior studies, although there were no significant differences in urine output, an age-related decrease in micturition frequency and an age-related increase in single voided volume were observed in both GK and Wistar rats. In the cystometric studies, although there were no significant differences in maximum detrusor pressure or bladder capacity, residual urine volume was significantly increased in the 70-week-old GK rats. In the functional studies, carbachol-induced contractility of the detrusor was significantly increased in GK rats of both age groups. The estimated $\mathrm{pA}_{2}$ values for atropine, pirenzepine, methoctramine, and 4-DAMP indicate that the carbachol-induced contractile response is mediated through the $\mathrm{M}_{3}$ receptor subtype in all groups. Furthermore, muscarinic $\mathrm{M}_{2}$ and $\mathrm{M}_{3}$ receptor mRNAs were 
significantly up regulated in the 70-week-old GK rat bladder.

Conclusion: Our data indicate that non-insulin-dependent diabetes induces alterations in the muscarinic receptor system, which may contribute to the development of diabetic cystopathy. 
Diabetic cystopathy, a form of urinary bladder dysfunction, is a major complication of diabetes, occurring in 25 to $83 \%$ of patients with diabetes mellitus. ${ }^{1,2}$ Kaplan et al. reported that classical diabetic cystopathy is not the most common urodynamic finding in patients with diabetes mellitus and voiding dysfunction, and in fact these patients present with variable Pathophysiological findings. ${ }^{3}$ The streptozotocin (STZ)-induced diabetic rat is the most commonly used and well-investigated experimental model for type 1 diabetes. ${ }^{4,5}$ Alterations of urinary bladder function are also seen in the STZ-induced diabetic rats. ${ }^{4,5}$ For example, increased urine output, frequent voiding, and atonic bladder are observed at least 2 weeks of diabetes induction in this animal model. ${ }^{6}$ However, only limited information is available about type 2 diabetic rat cystopathy. The Goto-Kakizaki (GK) rat represents a spontaneous non-insulin-dependent diabetes model. GK rats are produced from normal Wistar rats by repetition of selective breeding, and are a widely accepted genetically determined rodent model for human type 2 diabetes. $^{7,8}$ This genetic rat model is particularly relevant to human type 2 diabetes because defects in glucose-stimulated insulin secretion, peripheral insulin resistance, and hyperinsulinemia are seen as early as four weeks after birth, and later-occurring abnormalities include insulin secretion and modest hyperglycemia. $^{9}$

Furthermore, some reports have indicated age-related and diabetes-suffered duration-related alterations of bladder functions. ${ }^{10,11}$ To get more information on the detailed mechanisms of type 2 diabetes-induced alteration of bladder smooth muscles, 
we investigated bladder contraction using $\mathrm{KCl}$ and carbachol with subtype-selective muscarinic antagonists as well as muscarinic $\mathrm{M}_{2}$ and $\mathrm{M}_{3}$ receptor mRNAs expression levels by real-time polymerase chain reaction (PCR). 


\section{MATERIALS AND METHODS}

Animal model

All animal experiments were performed in accordance with the guidelines established by the Tottori University Committee for Animal Experimentation. Six-week-old male GK and Wistar rats were purchased from SLC (Shizuoka, Japan). All rats were kept under identical conditions, and had access to food and drinking water ad libitum. The rats were divided randomly into four groups $(n=6-8)$. Groups A and B consisted of 12-week-old Wistar and GK rats, respectively, while Groups C and D consisted of 70-week-old Wistar and GK rats, respectively. Upon reaching either 12 or 70 weeks of age, the rats were sacrificed with an overdose of pentobarbital (60 mg i.p.). Blood samples were collected from the vena cava, and isolated bladders were used in tissue bath experiments or frozen at $-80{ }^{\circ} \mathrm{C}$ for measurement of muscarinic $\mathrm{M}_{2}$ and $\mathrm{M}_{3}$ receptor mRNAs.

Serum glucose and serum insulin measurement

Serum glucose concentrations in the experimental rats were measured by the hexokinase method (Glucose CII , Wako Pure Chemical, Osaka, Japan), which was carried out according to the kit manufacturer's instructions in all groups. In Groups C and $\mathrm{D}$, the insulin concentrations were also measured by ELISA according to the manufacturer's instructions (Rat Insulin ELISA, Mercodia AB, Uppsala, Sweden).

\section{Voiding behavior studies}


Voiding behavior studies were performed according to methods used in our previous study $^{12}$ at 12 or 70 weeks of age in all groups. All rats received water ad libitum from the time they were initially placed in the cage. The parameters of the micturition reflex obtained were micturition frequency, total urine output, and single voided volume.

\section{Cystometric studies}

The cystometric studies were performed according to methods used in our previous report $^{12}$ at 12 or 70 weeks of age in all groups. The following parameters were evaluated according to our previous reports: bladder capacity, maximum detrusor pressure during voiding (Pdet), single-voided volume, and residual urine volume.

\section{Measurement of contractile force in the bladder}

Functional studies were conducted according to methods used in our previous reports. $^{12,13}$ Razor blades were used to cut uniform longitudinal strips of the posterior wall of the bladder dome $(1.5 \times 5 \mathrm{~mm})$. Changes in the tone of the strips were measured isometrically by means of force transducers, and the data were recorded on a personal computer (Macintosh G3, Apple Computer, Cupertino, CA, USA) with the use of the software program Chart version 3.6.9 and a PowerLab/16sp data acquisition system (AD Instruments, Castle Hill, NSW, Australia). Cumulative concentration-response curves to carbachol and $\mathrm{KCl}(100 \mathrm{mmol} / \mathrm{l})$ were constructed. Carbachol-induced contractile responses were measured cumulatively in the presence or 
absence of various concentrations of muscarinic receptor antagonists: pirenzepine (PRZ), methoctramine (MTR), 4-diphenylacetoxy-N-methylpiperidine methiodide (4-DAMP), and atropine (ATR). ${ }^{13}$ Antagonists were added 30 minutes prior to the administration of carbachol. After completion of a concentration-response curve, the tissue was washed until baseline force returned to the resting level, equilibrated for 30 minutes, and then the next consecutive concentration-response curve was constructed.

\section{Real-time PCR (quantification of muscarinic $M_{2}$ and $M_{3}$ receptor messenger}

\section{RNAs)}

To measure muscarinic receptor mRNAs on the detrusor, urothelium was carefully peeled under illuminated magnifiers in every rat (Otsuka, Tokyo, Japan). Muscarinic $\mathrm{M}_{2}$ and $\mathrm{M}_{3}$ receptor mRNAs in the experimental bladder dome were measured by real-time PCR methods according to our previous reports. ${ }^{12,} 13$ The RNA was purified by RNeasy® Fibrous Tissue Mini Kit (Qiagen, Valencia, CA, USA) according to the manufacturer's instructions. A reverse transcriptase (RT) mixture (28 $\mu \mathrm{l})$ containing $2 \mu \mathrm{g}$ of total RNA was made and incubated at $37{ }^{\circ} \mathrm{C}$ for 60 min according to a previously reported method. ${ }^{12,13}$ Five microliters of the mixture was used for real-time PCR, which was carried out with the use of a LightCycler thermal cycler system with a LightCycler-FastStart DNA Master Hybridization Probe (Roche Diagnostics, Tokyo, Japan) according to the manufacturer's instructions. ${ }^{14}$ The primers and probe sequences specific to the genes of muscarinicM $\mathrm{M}_{2}$ (accession number: NM 012527) and muscarinic $\mathrm{M}_{3}$ (accession number: NM 031016) receptors were used according to our 
previous reports. $^{12,13}$ The primer and probe of the $\beta$-actin (accession number: NM_031144) were used from the LightCycler-Primer/Probe Set (rat) (Roche Diagnostics). A total of $15 \mu \mathrm{l}$ of solution was used for the sample. PCR products were subjected to $2 \%$ agarose gel electrophoresis. The $\beta$-actin gene was used as the internal standard and analyzed by real-time PCR by using the same RT mixture.

\section{Data analysis}

The $\mathrm{EC}_{50}$ and $\mathrm{E}_{\max }$ values were obtained by a Macintosh computer (G3) located with Chart version 3.6.9 software and a PowerLab/16sp data acquisition system. The dose ratio was obtained from the ratio of $\mathrm{EC}_{50}$ values (the concentration of agonist that produces half-maximal contractile responses) for carbachol in the presence or absence of an antagonist. The pA2 values were obtained from Schild plots. ${ }^{15}$ Schild plots were constructed by plotting the log of (dose ratio -1) against the log of the molar concentration of the antagonist. The $\mathrm{EC}_{50}$ values were calculated as geometric means, whereas $E_{\max }$ values were calculated as arithmetic means. The expressions of muscarinic $\mathrm{M}_{2}$ and $\mathrm{M}_{3}$ receptor mRNAs were quantified according to the expressions of $\beta$-actin mRNAs in the experimental rat bladder domes. A statistical comparison of differences between groups was performed using analysis of variance and Fisher's multiple comparison tests. $\quad \mathrm{P}<0.05$ was regarded as the level of significance.

\section{Drugs and chemicals}

Carbachol, 4-DAMP, pirenzepine, and methoctramine were purchased from Sigma 
(St. Louis, MO, USA). Atropine was purchased from Wako Pure Chemical Industries (Osaka, Japan). All other chemicals were available commercially and of reagent grade. 


\section{RESULTS}

\section{General features of the experimental animals}

The data obtained regarding the general features and serum concentrations of insulin and glucose in the experimental animals are shown in Table 1 and Figure 1. The GK diabetic rats showed significantly small weight gain by the age of 10 weeks, as well as significantly small weight gain during the experimental period (Figure 1). There was no significant difference in the bladder weight between Groups A and B, or Groups C and D. However, the bladder / body weight ratio in Group D was markedly greater than that in Groups B and C. Significantly higher serum glucose levels were confirmed at the age of 12 weeks in the GK diabetic rats. Significantly higher serum glucose and lower serum insulin levels than those of the control rats were confirmed in the 70-week-old GK diabetic rats.

\section{Voiding behavior studies}

Results of voiding behavior studies for the experimental animals are shown in Table 2. These studies revealed no significant differences in urine production, micturition frequency, or single voided volume between the GK and control rats at either age. Although urine production was similar in all groups, age-related alterations in micturition frequency and single voided volume were observed. The older rats (Groups C and D) showed significant decreases in micturition frequency and significant increases in single voided volume. 


\section{Cystometric studies}

The results of the cystometric studies are also shown in Table 3. In these studies, although single voided volume in Groups $\mathrm{C}$ and $\mathrm{D}$ tended to be larger, there were no significant differences in Pdet and single voided volume between any of the groups. However, the residual urine volume in Group D was markedly greater than those in Groups B and C.

\section{Measurement of contractile responses to carbachol and $100 \mathrm{mM} \mathrm{KCl}$}

The $E_{\max }$ values for the contractile responses of the longitudinal muscles to carbachol and $\mathrm{KCl}(100 \mathrm{mM})$ were determined (Table 4). Diabetes-induced detrusor hyperreactivity and age-related hyporeactivity by carbachol was observed in these experimental rats when normalized by $100 \mathrm{mmol} \mathrm{KCl}$. However, there were no significant differences in the $\mathrm{EC}_{50}$ values with respect to carbachol between any of the groups. The $\mathrm{pA}_{2}$ values and slopes of the Schild plots for these muscarinic receptor antagonists in the rat detrusor are shown in Table 5. The $\mathrm{pA}_{2}$ values calculated for series of muscarinic antagonists were similar in all groups and were rank-ordered as ATR $>$ 4-DAMP $>$ MTR $>$ PRZ. The slopes of the Schild plots for these muscarinic receptor antagonists were similar between groups.

These data suggest that in control bladder smooth muscle, contractile responses induced by carbachol are mediated through the muscarinic $\mathrm{M}_{3}$ receptor subtype, and that carbachol does not alter contractile systems according to age or the presence of diabetes. 


\section{Measurement of muscarinic $\mathbf{M}_{2}$ and $\mathbf{M}_{3}$ receptor mRNAs in the rat bladder dome}

Table 6 shows the expressions of muscarinic $\mathrm{M}_{2}$ and $\mathrm{M}_{3}$ receptor mRNAs in the bladder dome. Although the expression levels of muscarinic $\mathrm{M}_{2}$ and $\mathrm{M}_{3}$ receptor mRNAs were similar in the younger groups, those of the older GK rats were significantly higher than those of age-matched controls. Furthermore, the control group (Group A) had higher expression levels of the muscarinic $\mathrm{M}_{3}$ than the muscarinic $M_{2}$ receptor mRNAs; in all groups, the expression level of the muscarinic $M_{3}$ receptor mRNAs was approximately 2-3 times higher than that of the muscarinic $\mathrm{M}_{2}$ receptor mRNAs. 


\section{DISCUSSION}

Latifpour and associates demonstrated that significant up-regulation of the expressions of muscarinic receptors and hyper-contractility induced by a muscarinic receptor agonist, carbachol, in diabetic rat bladder dome., 5 Interestingly, those reports did not demonstrate significant alterations of $\mathrm{EC}_{50}$ values for carbachol and of $\mathrm{K}_{\mathrm{i}}$ values of $\left[{ }^{3} \mathrm{H}\right] \mathrm{QNB}$ for muscarinic receptor subtype-selective antagonists. Those findings indicated that diabetes-induced alterations of the muscarinic receptor system in the bladder are quantitative rather than qualitative. Tong and associates reported that STZ-induced diabetes increases mRNA and protein expressions of $M_{2}$ and $M_{3}$ muscarinic receptors in the urothelium as well as in the muscle layer. ${ }^{16,17}$ In our recent studies, analyses of diabetes-induced alterations in the bladder using pharmacological, biochemical, and biological methods supported their findings. ${ }^{12,13}$ Recently, Daneshgari and associates reported that diabetic bladders may undergo a transition from a compensated to a decompensated state and that this transition in the STZ rat model may begin 9 to 12 weeks after induction. ${ }^{11}$ Thus, the STZ rat model of type 1 diabetes has been well investigated and characterized. However, only limited information is available for bladder dysfunction in type 2 diabetic models. Using 32-week-old female GK rats, Miyamae et al. reported that damage to the autonomic nervous system and peripheral nerves in the bladder causes a decrease in acetylcholine release during bladder contractions, which may be related to voiding dysfunctions in diabetes mellitus. ${ }^{18}$ Yono et al. reported age-related alterations in the biochemical and functional properties of the bladder in type 2 diabetic GK rats. In their reports, the maximum 
contractile responses to carbachol and ATP, and the release of acetylcholine induced by field stimulation, were similar in bladders from GK and control rats until 8 weeks of age; however, at the ages of 16 and 32 weeks, GK rats had increased contractile responses to carbachol and levels of ATP, along with decreased release of acetylcholine, compared to controls. ${ }^{19}$

Previously, we reported that diabetes induces an increase in maximum detrusor pressure during voiding; this increase occurred by urethral dysfunction associated with diabetic neuropathy. $\frac{12}{2}$ In this study, we demonstrated similar tendency of Pdet in GK rats; Pdet in 70-week-old GK rats (Group D) tended to be higher than that in age-matched Wistar rats (Group C). However, there was no significant difference between Groups C and D. It may be due to great variation of the data presented. In the present study, we also demonstrated the hypercontractility of detrusor smooth muscle to carbachol in GK rats at both ages compared to age-matched control rats. These data are similar to those for STZ-induced diabetic rats previously reported. ${ }^{12,13}$ We also demonstrated that 70-week-old GK diabetic rats had significantly increased residual urine volume compared to both age-matched control rats and 12-week-old GK rats. These data are particularly interesting, because early-stage STZ-induced diabetic rats and late-stage GK rats showed similar patterns of cystopathy. To clarify the mechanisms underlying type 2 diabetic cystopathy, we undertook pharmacological and biological examinations. To confirm these putative changes in the muscarinic receptor system, we calculated the $\mathrm{pA}_{2}$ values and their slopes using four subtype nonselective and selective muscarinic alterations. There were no significant differences in the $\mathrm{pA}_{2}$ 
values and slopes between diabetic and non-diabetic rats for any of the muscarinic receptor antagonists used in the present study. The results of the $\mathrm{pA}_{2}$ calculations for this series of muscarinic antagonists were similar in all groups, and the rank order of the values was: ATR $>4$-DAMP $>$ MTR $>$ PRZ. These findings indicate that alteration of the contractile response via the muscarinic $\mathrm{M}_{3}$ receptor subtype is not due to changes in muscarinic receptor affinity in diabetic rat detrusor. Rather, such changes appear to be the result of quantitative rather than qualitative changes in the muscarinic receptor system. Mutoh and associates previously reported that the rank order of $\mathrm{pA}_{2}$ values for these muscarinic antagonists was ATR $>4$-DAMP $>$ PRZ $>$ MTR in the rabbit bladder dome, ${ }^{20}$ and we also previously reported that the rank order of $\mathrm{pA}_{2}$ values for these muscarinic antagonists in the rat bladder smooth muscle was ATR $>4$-DAMP $>$ MTR > PRZ. ${ }^{13}$ Regarding the role of muscarinic receptors, these data suggest that the rat detrusor undergoes contractions via the muscarinic $\mathrm{M}_{3}$ receptor subtype in both younger and older GK rats.

To elucidate the mechanisms underlying diabetes-induced hypercontractility to carbachol of the rat detrusor, we measured the expression levels of muscarinic $\mathrm{M}_{2}$ and $\mathrm{M}_{3}$ receptor mRNAs using real-time PCR. The results showed that the mRNA levels of both muscarinic $\mathrm{M}_{2}$ and $\mathrm{M}_{3}$ receptors were increased under diabetic conditions at 70 weeks, as compared to age-matched controls. Based on our previous and present data, it appears likely that the overexpressions of muscarinic $\mathrm{M}_{2}$ and $\mathrm{M}_{3}$ receptor mRNAs are related to the hypercontractility of detrusor in 70 -week-old diabetic rats. ${ }^{12,13}$ The possible mechanisms of this up-regulation of muscarinic receptors are explained due to 
decrease in cholinergic nerve density, ${ }_{2}^{18}$ or due to defective neurotransmitter release $\underline{\text { mechanism. }} . \underline{21}$ Miyamae et al. reported decrease in cholinergic nerve density in GK rats, $\underline{18}$ while Tong et al. reported defective neurotransmitter release mechanism in two-week-old STZ induced diabetic rats. ${ }^{21}$ The diabetes-associated neuropathy may inhibit the release of acetylcholine from cholinergic nerves, in turn inducing the overexpression of muscarinic receptors in the diabetic detrusor. ${ }^{18,} 19$ Such overexpression may enhance signaling downstream of these receptors and may increase detrusor contraction, according to the results of the present organ bath study.

In conclusion, our data indicate that non-insulin-dependent diabetes induces alterations of the muscarinic receptor system that may contribute to the development of diabetic cystopathy. 


\section{References}

1. Faerman I, Maler M, Jadzinsky M, Alvarez E, Fox D, Zilbervarg J, et al. Asymptomatic neurogenic bladder in juvenile diabetics. Diabetologia, 1971; 7: 168.

2. Bradley WE. Diagnosis of urinary bladder dysfunction in diabetes mellitus. Ann Intern Med 1980; 92: 323.

3. Kaplan SA, Te AE and Blaivas JG. Urodynamic findings in patients with diabetic cystopathy. J Urol 1995; 153: 342.

4. Latifpour J, Gousse A, Kondo S, Morita T and Weiss RM. Effects of experimental diabetes on biochemical and functional characteristics of bladder muscarinic receptors. $\mathrm{J}$ Pharmacol Exp Ther 1989; 248: 81.

5. Fukomoto Y, Yoshida M, Weiss RM and Latifpour J. Reversibility of diabetes- and diuresis-induced alterations in rat bladder dome muscarinic receptors. Diabetes 1994; 43: 819.

6. Nakamura I. Takahashi I and Miyagawa I. The alterations of norepinephrine and acetylcholine concentrations in immature rat urinary bladder caused by streptozotocin-induced diabetes. J Urol 1992; 148: 423. 
7. Goto $\mathrm{Y}$ and Kakizaki $\mathrm{M}$. The spontaneous-diabetes rat: A model of noninsulin-dependent diabetes mellitus. Proc Japn Acad 1981; 57: 381.

8. Goto Y, Kakizaki M and Masaki N. Production of spontaneous diabetic rats by repetition of selective breeding. Tohoku J Exp Med 1976; 119: 85-90.

9. Murakawa Y, Zhang W, Pierson CR, Brismar T, Ostenson CG, Efendic S, et al. Impaired glucose tolerance and insulinopia in the GK-rat causes peripheral neuropathy. Diabetes Metab Res Rev 2002; 18: 473.

10. Latifpour J, Kondo S, O'Hollaren B, Morita T and Weiss RM. Autonomic receptors in urinary tract: sex and age differences. J Pharmacol Exp Ther_1990; 253: 661.

11. Daneshgari F, Liu G and Imrey PB. Time dependent changes in diabetic cystopathy in rats include compensated and decompensated bladder function. J Urol 2006; 176: 380.

12. Saito M, Kinoshita Y, Satoh I, Shinbori C, Suzuki, H, Yamada M, et al. Ability of cyclohexenonic long-chain fatty alcohol to reverse diabetes-induced cystopathy in the rat. Eur Urol 2007; 51; 479.

13. Kazuyama E, Saito M, Okada S and Satoh K. Ability of cyclohexenonic long-chain 
fatty alcohol to ameliorates diabetes-induced cystopathy in the rat. Pharmacology 2007; 81: 137.

14. Wittwer CT, Ririe KM, Andrew RV, David,DA, Gundry RA and Balis UJ. The LightCycler: a microvolume multisample fluorometer with rapid temperature control. Biotechniques 1997; 22:176.

15. Arunlakshana $\mathrm{O}$ and Schild HO. Some quantitative uses of drug antagonists. Br J Pharmacol 1959; 14: 48.

16. Tong Y-C, Cheng J-T and Hsu C-T. Alterations of M2-muscarinic receptor protein and mRNA expression in the urothelium and muscle layer of the streptozotocin-induced diabetic rat urinary bladder. Neurosci Lett 2006; 406: 216-221.

17. Cheng J-T, Yu B-C and Tong Y-C. Changes of M3-muscarinic receptor protein and mRNA expressions in the bladder urothelium and muscle layer of streptozotocin-induced diabetic rats. Neurosci Lett 2007; 423: 1.

18. Miyamae K, Yoshida M, Inadome A, Murakami S, Otani M, Iwashita H, et al. Acetylcholine release from urinary bladder smooth muscles of non-insulin-dependent diabetic rats. Urol Int 2004; 73: 74. 
19. Yono M, Latifpour J, Yoshida M and Ueda S. Age-related alterations in the biochemical and functional properties of the bladder in type 2 diabetic GK rats. J. Recept Signal Transduct Res 2005; 25: 147.

20. Mutoh S, Latifpour J, Saito M and Weiss RM. Evidence for the presence of regional differences in the subtype specificity of muscarinic receptors in rabbit lower urinary tract. J. Urol. 1997; 157: 717.

21. Tong Y-C, Hung Y-C, Lin S-N and Cheng J-T. Alterations in urinary bladder synaptosomal neurotransmitter concentrations in two-week streptozotocin-induced diabetic rats. Neurosci Lett 1996; 206:165. 


\section{Figure legends}

Figure 1. Body weight in GK and Wistar rats.

Data are shown as mean \pm SEM of six to eight separated determinations in each group.

*)significantly different from the same age of Wistar rats. 


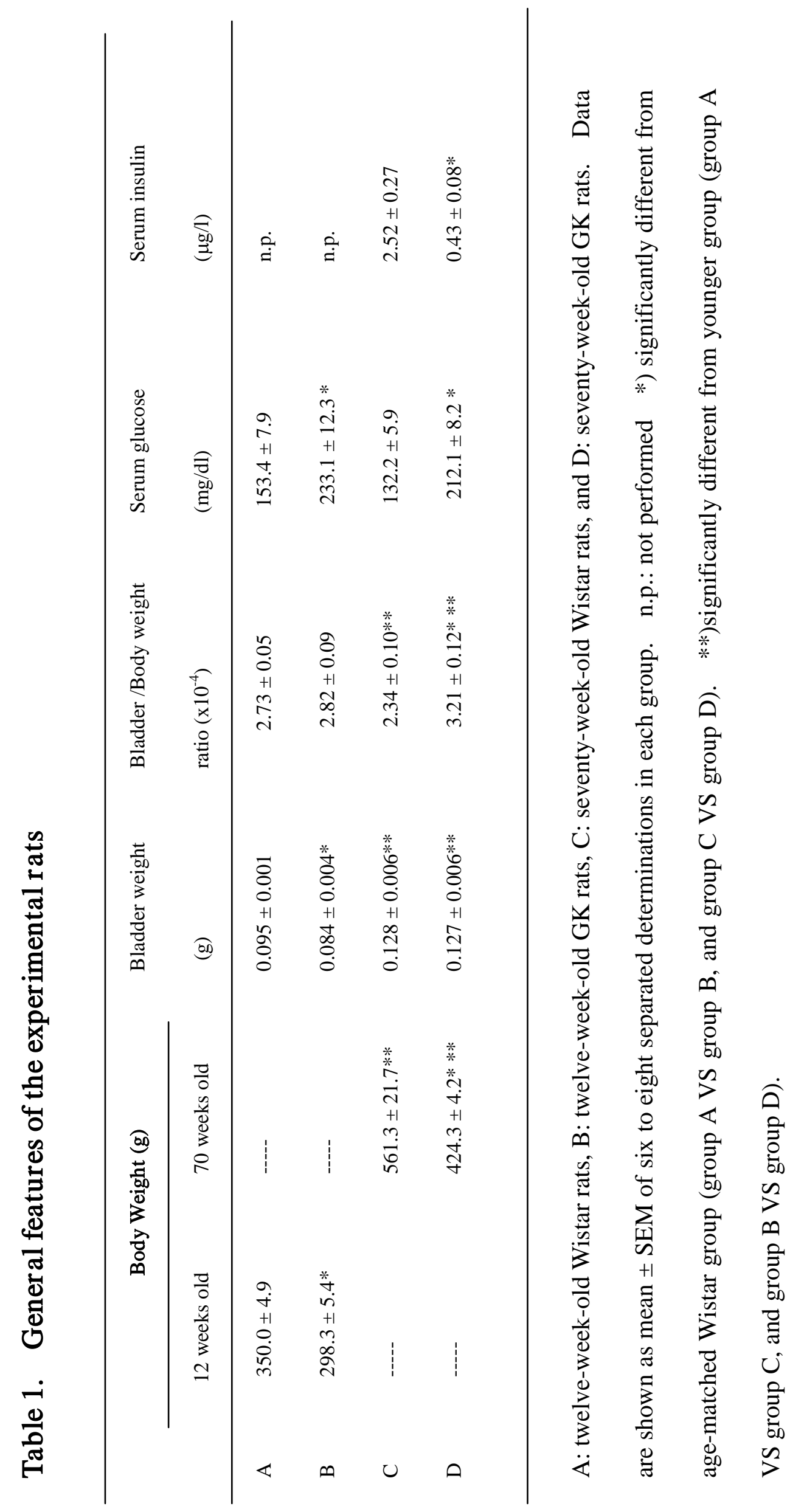




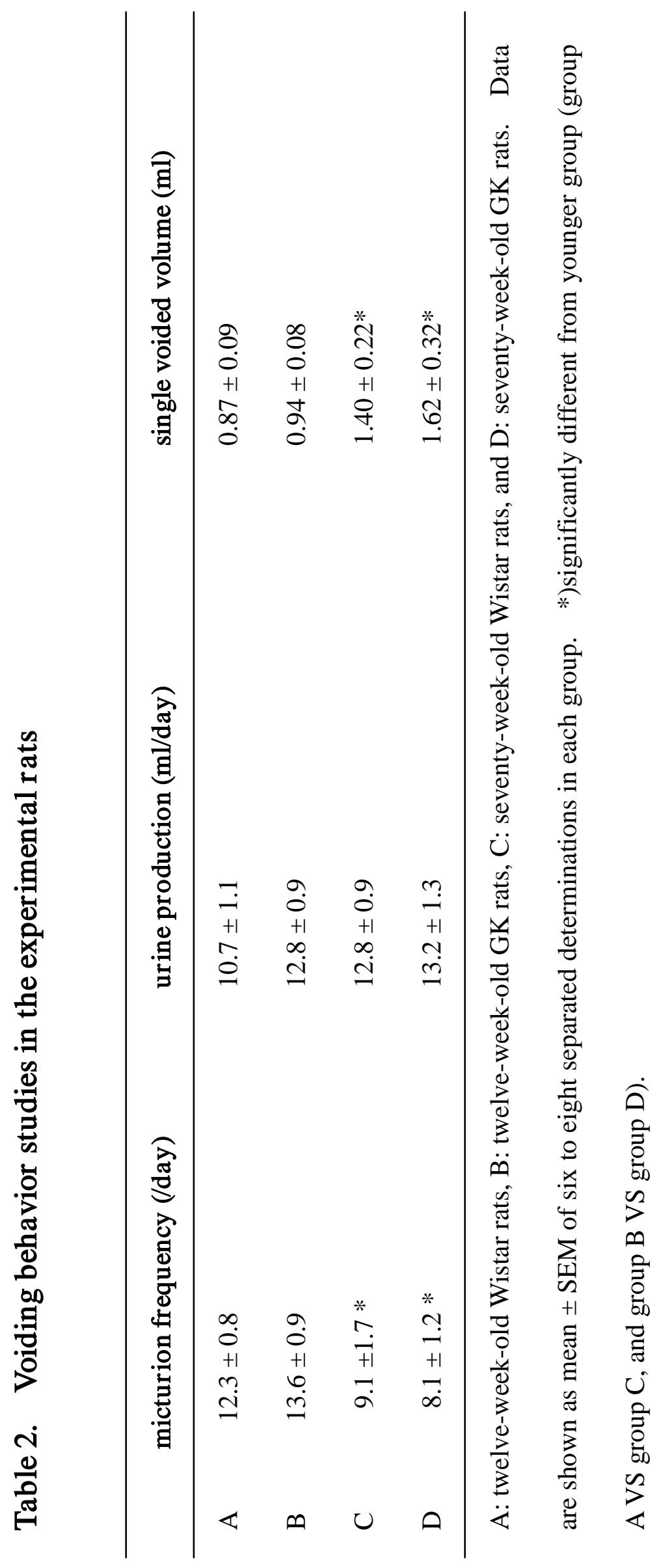




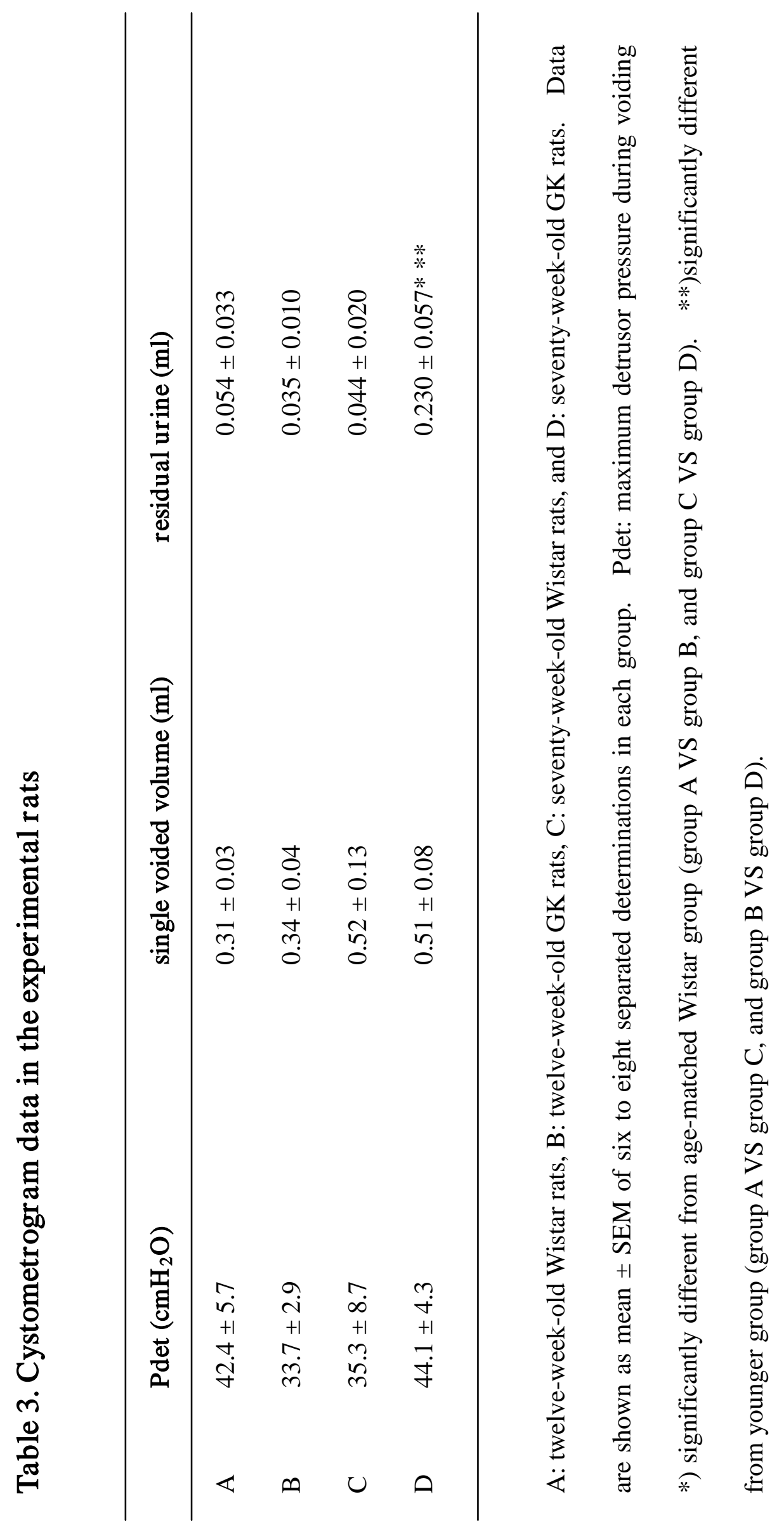




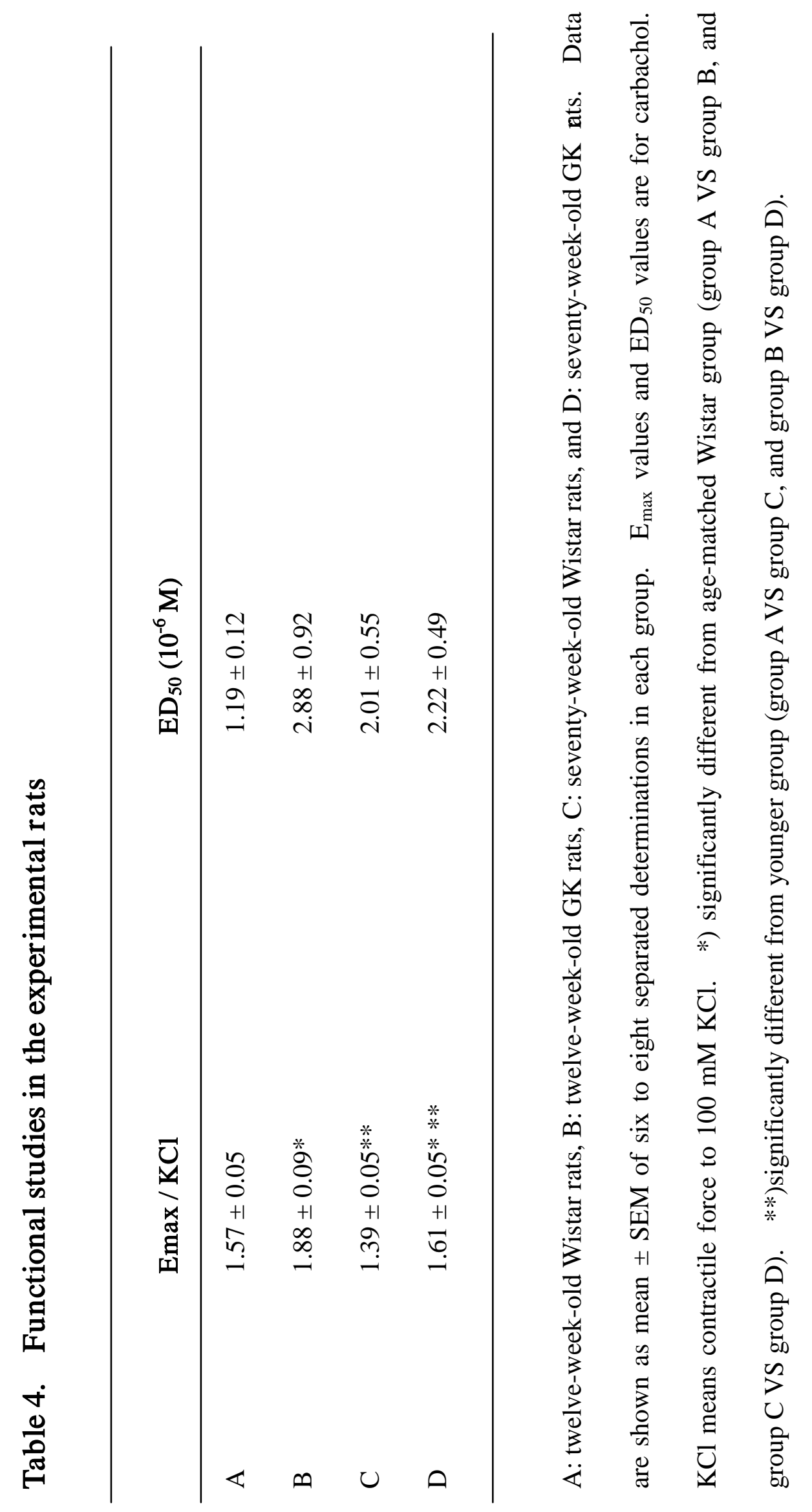




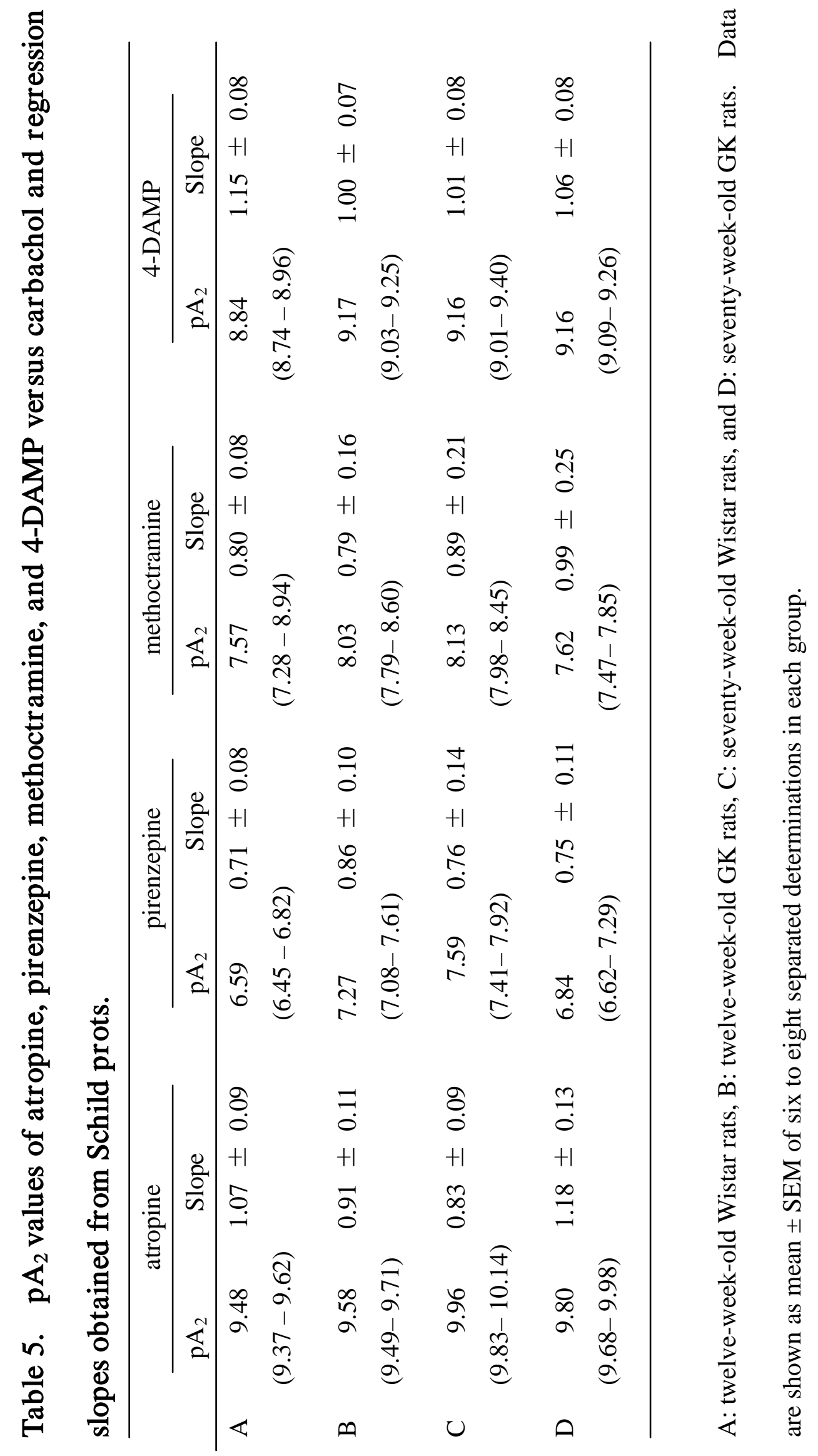




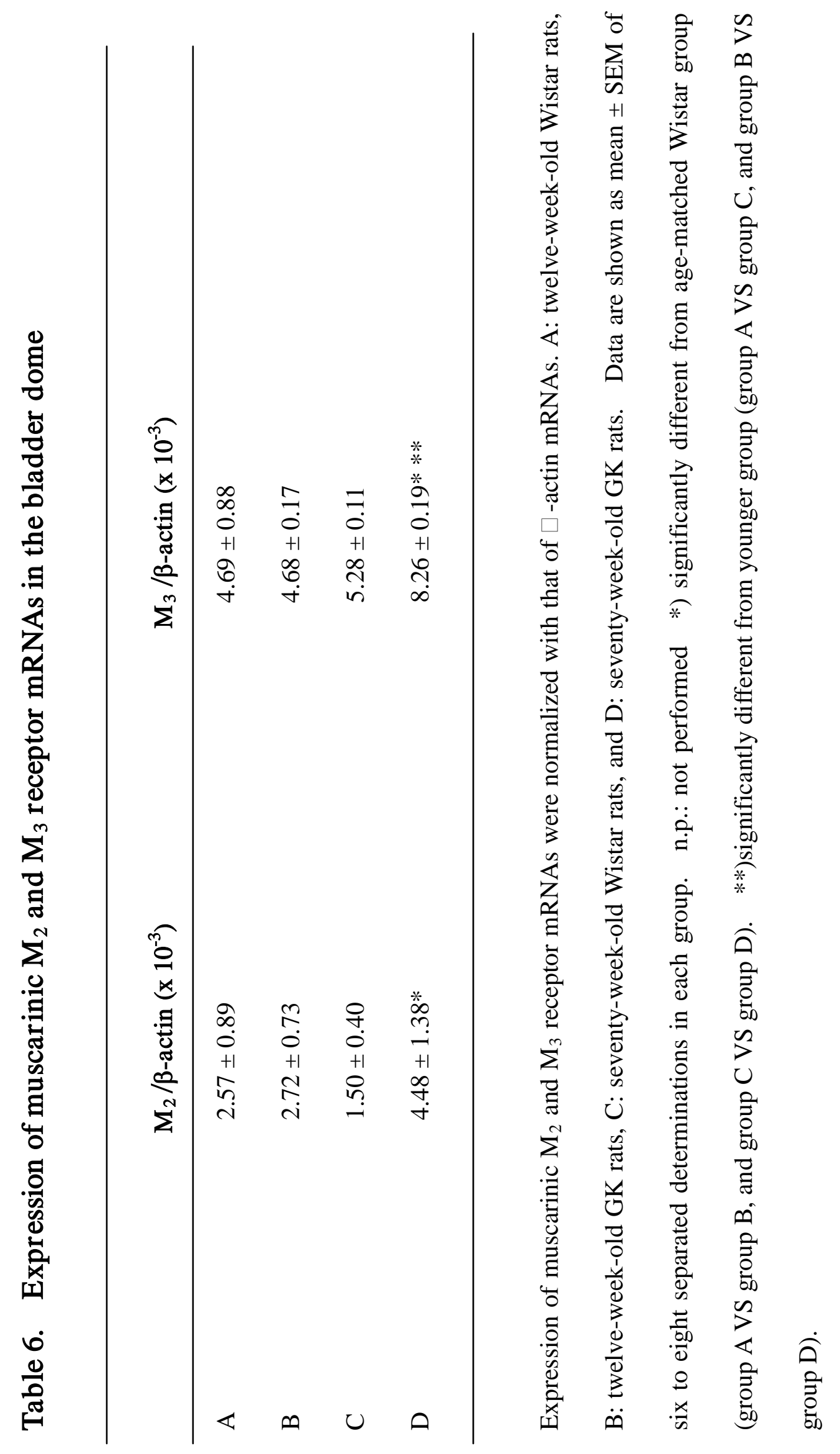




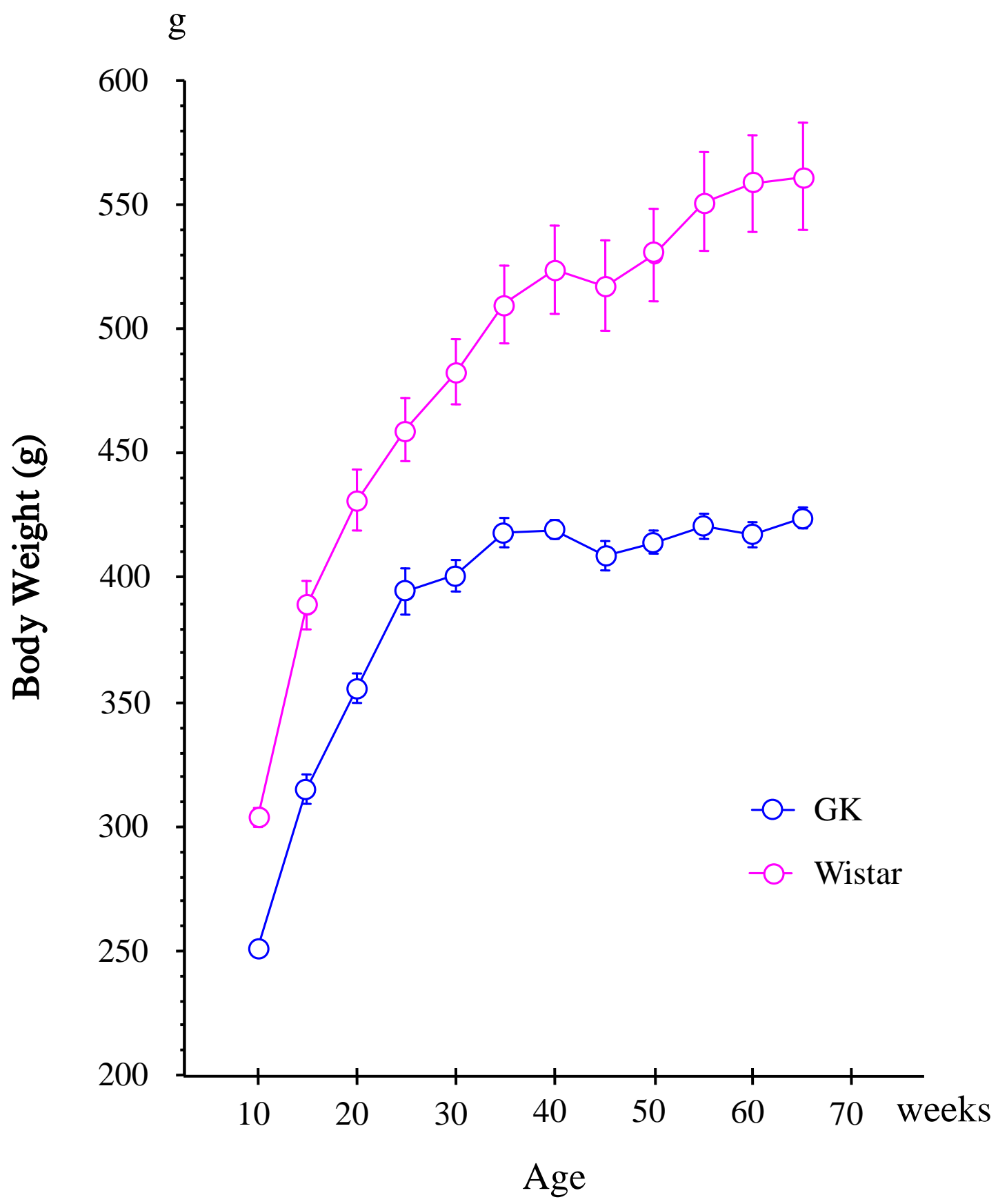

